\section{The Effect of Manufacturing Methods and Different Shapes on the Release Pattern of Diclofenac Sodium Matrix Tablet}

\author{
Tajnin Ahmed ${ }^{1}$, Muhammad Shahidul Islam ${ }^{2 *}$, Tasnuva Haque ${ }^{1}$, \\ Mohammad Abusyed ${ }^{3}$ \\ ${ }^{1}$ Department of Pharmacy, Stamford University Bangladesh, 51 \\ Siddeswari Road, Dhaka-1217, Bangladesh \\ ${ }^{2}$ Department of Pharmacy, East West University, Dhaka, Bangladesh \\ ${ }^{3}$ Product Management Department, UniMed UniHealth Pharma Limited, \\ Dhaka. Banaladesh
}

Received- 5 February 2009

\author{
*Corresponding Author \\ Muhammad Shahidul Islam \\ Lecturer, Department of Pharmacy \\ Stamford University Bangladesh \\ Contact no.: +88-01819559297 \\ E-mail: shahidul.pharmdu@yahoo.com
}

\begin{abstract}
In the present study sustained release diclofenac sodium matrix tablets were prepared using Kollidon SR polymer. Hydroxypropyl methylcellulose (HPMC $15 \mathrm{cps}$ ) and poly ethylene glycol (PEG-600) polymers respectively were used in formulating tablets prepared by direct compression and wet granulation methods. The polymers were used to explore the release pattern of the drug into the dissolution media. The tablets were also prepared in various shapes (caplet oval, round oval and flat oval). A comparatively higher release rate of drug was obtained from the polymer HPMC $15 \mathrm{cps}$ at $10 \%$ concentration for directly compressed matrix tablet than those containing $20 \%$ of HPMC after a definite period of time. In wet granulation process, $10 \%$ PEG-600 containing tablets showed a better release than those containing 20\% PEG. The drug release was also found to be sustained in case of wet granulation method than that of the direct compression method. Again the caplet shaped tablets in case of direct compression method showed better release rate of drug than those of the round oval and flat oval shaped tablets. Thus the result of this study shows that the proper selection of the percentage of polymer and the suitable shape of tablet and proper manufacturing method can provide a greater opportunity in designing sustained release dosage forms.
\end{abstract}

Key words: Matrix tablet, release pattern, direct compression, wet granulation, PEG 600, Kollidon SR.

\title{
INTRODUCTION
}

One of the most commonly used methods of modulating tablet drug release is to include it in a matrix system. The classification of matrix systems is based on matrix structure, release kinetics, controlled release properties (diffusion, erosion, swelling), and the chemical nature and properties of employed materials (Salsa et al., 1997). Matrix systems are usually classified in 3 main groups: hydrophilic, inert, and lipidic.HPMC is a semisynthetic nonionic cellulose ether, which is widely used in controlled-release dosage forms because of its nontoxic nature, its capacity to accommodate high levels of drug loading, and its non $\mathrm{pH}$ dependence. Drug release from hydrophilic matrix tablets is controlled by a hydrated viscous layer formed at the tablet periphery; this gel layer acts as a barrier to drug release (Amaral et al., 2001). Drug release from HPMC matrix tablets may be affected by several variables, such as tablet shape, size and surface area (Colombo et al., 1992). Once a hydrophilic matrix tablet is developed and the release profile is established with a certain tablet shape, there is reluctance to modify the product geometry. This is particularly true for drugs at the extremes of dose or solubility, for which the drug release is mainly controlled via diffusion or erosion. A previous study examined the release of highly water soluble drugs from HPMC matrices and demonstrated that when surface area to volume ratio (SA/V) is held constant, the drug release profiles are similar regardless of the tablet shape (round or oval) (Reynold et al., 2004). Preparation of drug-embedded matrix tablet that involves the direct compression of a blend of drug, retardant material and additives is one of the least complicated approaches for the manufacture of sustained release dosage forms. Plastic matrix systems, due to their chemical inertness and drug-embedding ability, have been widely used for sustaining the release of drugs (Lordi et al., 1990). Kollidon SR is one of the recently developed matrix forming agents with plastic behaviour. Chemically, Kollidon SR is polyvinyl acetate and polyvinyl pyrrolidone based matrix retarding agent particularly suitable for the manufacture of $\mathrm{pH}$ independent sustained release matrix tablets. Polyvinyl acetate is a very plastic material that produces a coherent mass even under low compression force. When the tablets prepared with Kollidon SR are introduced into gastric or intestinal fluid, the water soluble polyvinylpyrrolidone is leached out to form pores through which the active ingredients slowly diffuses outwards in a 
controlled and pre-determined fashion. Kollidon SR contains no ionic groups which render them inert to the drug molecule. Its high flowability, low reposition angle and excellent compressibility a characteristic endows the tablets with desired hardness and low friability while simultaneously reducing the process variables and processing cost (BASF, 1999). The purpose of the present study was to find out the release rate retardation action of three polymers (Kollidon SR, HPMC 15 cps and PEG 600), the effect of manufacturing methods and the effect of tablet shapes on the release pattern of diclofenac sodium from the prepared matrix tablets.

\section{MATERIALS AND METHODS}

Diclofenac sodium (DS) was a gift sample from Beximco Pharmaceuticals Limited. Reagents: Methocel (HPMC $15 \mathrm{cps}$ ) and PEG 600 were collected from Colorcon, USA. Kollidon SR, lactose and maize starch were gift samples from Loba chemie Pvt. Ltd, India. Magnesium stearate and talc were obtained from Hanna chemical Ltd. (Japan). All the other reagents employed were of pharmaceutical grade.

\section{Preparation of DS matrix tablets by direct compression technique}

DS was weighed out (Denver electronic balance, Switzerland) and all the excipients (according to Table 1) were mixed thoroughly for uniform mixing and then it was sieved though 60 mesh sized screen. After proper mixing the mixed materials were taken for direct compression in a tablet punch machine (Jaguar, India) using hand pressure. Tablets of various shapes were prepared using different sets of die and punches. Formulations prepared in direct compression process were coded as FDC-1 and FDC-2.

\section{Preparation of DS matrix tablets by wet granulation technique}

Lactose and maize starch were dissolved in ethanol to produce the binder solution. Separately, DS and polymers were mixed homogenously. Then the binder solution was added to the drug polymer powder mix gradually and a wet mass was prepared. Again the wet mass was passed through a sieve of desired mesh size, granules were prepared and were blended with talc and magnesium stearate. Finally, the mixture was taken in a tablet punch machine. Tablets of various shapes were produced by wet granulation method (Table 1). Formulations prepared in wet granulation process were coded as FWG-1 and FWG-2.

Table 1: Formulations of DS matrix tablets prepared by direct compression and wet granulation methods

\begin{tabular}{|c|c|c|c|c|}
\hline \multirow{2}{*}{$\begin{array}{c}\text { Ingredients } \\
\text { mg/tablet) }\end{array}$} & \multicolumn{4}{|c|}{ Formulation Code } \\
\cline { 2 - 5 } & FDC-1 & FDC-2 & FWG-1 & FWG-2 \\
\cline { 2 - 5 } & 100 & 100 & 100 & 100 \\
\hline Diclofenac Sodium & 75 & 75 & 65 & 65 \\
\hline Kollidon SR & 26 & 52 & - & - \\
\hline HPMC 15 cps & - & - & 26 & 52 \\
\hline PEG 600 & 25 & 10 & 30 & 20 \\
\hline Maize Starch & 25 & 15 & 30 & 4 \\
\hline Lactose & 4 & 4 & 4 & 4 \\
\hline Talc & 5 & 4 & 5 & 260 \\
\hline Magnesium Stearate & 260 & 260 & 260 & 4 \\
\hline Total weight/Tablet & &
\end{tabular}

\section{In-vitro Dissolution study}

In-vitro Dissolution study was performed in order to observe the release kinetics of drugs containing different polymers at different concentrations and the effect of different shapes on the release of the drug from tablet and also the effect of manufacturing method. Dissolution studies were conducted according to the USP method (USP XXII) using apparatus-I (Erweka, Germany) i.e. a cylindrical basket in the metallic drive shaft instead of paddle type string shaft and at $50 \mathrm{rpm}$. The temperature was kept constant at $37 \pm 0.5^{\circ} \mathrm{C} .1000 \mathrm{ml}$ of the dissolution medium $(\mathrm{pH} 6.5$ phosphate buffer) was placed in the vessel, which, has been previously immersed in the constant temperature water bath and the dissolution medium was allowed to come to the temperature of $37 \pm 0.5^{\circ} \mathrm{C}$. At every one-hour interval $10 \mathrm{ml}$ solution was collected and analyzed by UV 
spectrophotometer at $277 \mathrm{~nm}$ (Shimadzu, Japan). The drug concentration was calculated with the help of straight-line equation obtained from the respective standard curves. For each sampling, the volume was adjusted with $10 \mathrm{ml}$ fresh dissolution media. Drug dissolved at specified time period was plotted as percent release versus time (hours) curve.

Kinetic modeling of drug release. After completing in vitro dissolution of all the batches for eight hours, the data were treated with zero order equation (Donbrow et al., 1980) and Higuchi equation (Higuchi, 1961):

$M_{t}=M_{0}+k_{0} t$

$M t=M_{0}-k_{H} t^{1 / 2}$

In these equations, $M_{t}$ is the cumulative amount of drug released at any specified time $(\mathrm{t})$ and $\mathrm{M}_{0}$ is the dose of the drug incorporated in the delivery system. $\mathrm{k}_{0}$ and $\mathrm{k}_{\mathrm{H}}$ are rate constants for zero order and Higuchi model respectively. These models failed to explain drug release mechanism due to swelling (upon hydration) along with gradual erosion of the matrix. Therefore the dissolution data were also fitted to well-known Korsmeyer kinetic equation to ascertain the mechanism of drug release (Korsmeyer et al., 1983 and Peppas, 1985)

$\log \left(M_{t} / M_{\infty}\right)=\log k+$ nlogt......

Where $M_{\infty}$ is the amount of drug release after infinite time; $k$ is the release rate constant which considers structural and geometric characteristics of the tablet; and $\mathrm{n}$ is the diffusional exponent or release exponent; indicative of the mechanism of drug release. For a tablet having cylindrical shape, when $\mathrm{n}$ is below 0.45 , the Fickian diffusion phenomenon dominates, and $\mathrm{n}$ between 0.45 and 0.89 is an anomalous transport (non-Fickian diffusion), often termed as first-order release. After the $\mathrm{n}$ value reaches 0.89 and above, the release can be characterized by case II and super case II transport, which means the drug release rate does not change over time and the release is characterized by zero order. In this case, the drug release is dominated by the erosion and swelling of the polymer.

\section{RESULTS AND DISCUSSION}

Diclofenac sodium matrix tablets were prepared by using some polymers such as Kollidon SR, poly ethylene glycol (PEG), and hydroxypropyl methylcellulose (HPMC) in different percentages by using the two tabletting methods and at different shapes. Polymers were used as release modifier. The dissolution studies of all batches of tablet were carried out by following the USP methods (USP XXII). The results and discussions were in the following section:

\section{In vitro DS release from the matrix tablets: Effect of method of manufacturing}

DS matrix tablets were manufactured by two methods - direct compression and wet granulation. In direct compression method, DS was mixed with hydroxypropyl methylcellulose (HPMC $15 \mathrm{cps}$ ) with a fixed percentage (30\%) of Kollidon SR. The percentages of HPMC were $10 \%$ and $20 \%$. In wet granulation method, ethylene glycol (PEG 600) was used as the polymer at the percentages of $10 \%$ and $20 \%$ with the fixed percentage $(25 \%)$ of Kollidon SR. Here different manufacturing methods were used to monitor which method was most suitable for the sustained release dosage forms. It was found that in case of formulations made by direct compression method, the formulation FDC-1 (containing 30\% Kollidon SR and 10\% HPMC 15cps) showed $90.136 \%$ release of DS after 8 hours. On the other hand, FDC-2 that contained 30\% Kollidon SR and 20\% HPMC 15 cps showed $83.294 \%$ release of the drug at the same duration. Whereas, FWG-1 (contained with 25\% Kollidon SR and 10\% PEG 600) and FWG-2 (contained with 25\% Kollidon SR and 20\% PEG 600 ) release about $77.92 \%$ and $73.14 \%$ DS at 8 hours. Comparing the four formulations prepared in two methods, it can be stated that tablets prepared with direct compression method showed less sustained release upto 8 hours than that of wet granulation methods. Doubling the concentration of polymer in both methods, the release rate was decreased by $6.85 \%$ (in direct compression method) and $4.78 \%$ (in wet granulation method). Another reason may be included that, in direct compression method, the Kollidon SR used in higher concentration $(30 \%)$ than that of wet granulation method (25\%) Again in direct compression method HPMC $15 \mathrm{cps}$ was used as the copolymer and in wet granulation method PEG 600 was used as copolymer.

\section{In vitro DS release from the matrix tablets: Effect of tablet shapes}

DS matrix tablet was prepared as $260 \mathrm{mg}$ tablet by using by using different shaped punches. The shape of tablets was changed to monitor the effect of shape on the released rate of the drug. Here the direct compression method was used for all the three shapes of the tablets. 


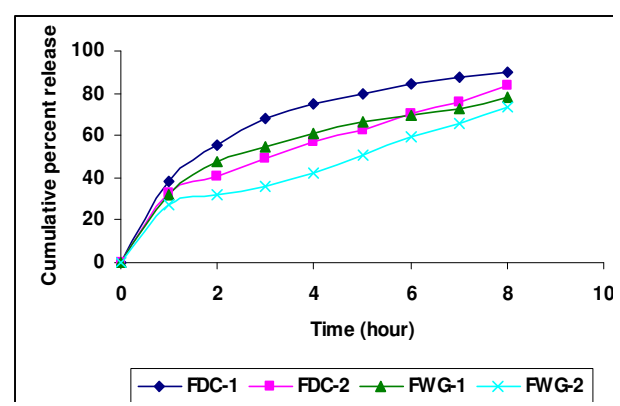

Figure 1: Cumulative percent release vs. time curve of different formulations prepared by direct compression (FDC-1 and FDC-2) and wet granulation (FWG-1 and FWG-2) methods.

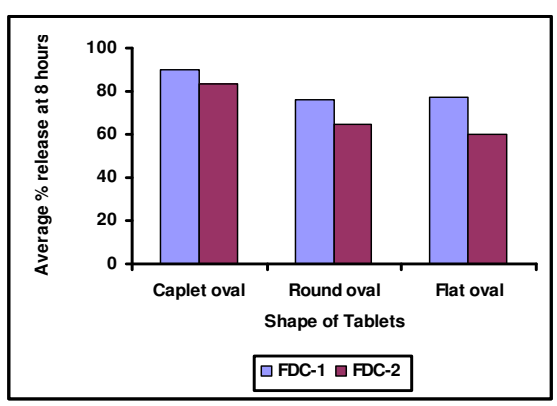

Figure 2: Comparison of average \% release of different shapes of tablets prepared by direct compression method.

Figure 2 shows the release profiles of DS matrix tablets which were manufactured in different shapes by direct compression method. The total percent release of DS from three different shapes, that is, caplet oval, round oval and flat oval tablets (FDC-1) was 90.136, 76.33 and $77.49 \%$ respectively. Whereas, FDC-2 showed $83.294,64.95$ and $60.11 \%$ release of DS from the previously stated shaped tablets respectively. In the figure it is observed that a higher percentage of drugs were release from the caplet oval shaped directly compressed matrix tablet compared with the other shapes. The table 3 showed different kinetic parameters of directly compressible tablets and the tablets prepared by wet granulation method. Here the $R^{2}$ value 0.982 and 0.979 (From Higuchi plot) indicates that the release pattern of drug from FDC-1 and FDC-2 best fitted with the Higuchi release order (0.982 and 0.979). The best fitted plot indicates that the release of DS from the matrix tablet was mainly by diffusion through the pore formed on the surface of the tablet due to dissolution process. FDC-1 and FDC-2 were also fitted to Korsmeyer plot as they had the $R^{2}$ values of 0.977 and 0.978 respectively. Again the release exponent $(n)$ value of FDC-1 was 0.734 and that of FDC-2 was 0.761 indicates that the release of the drug from both the tablets was non-Fickian i.e. release is by diffusion followed by erosion mechanism. Similarly, FWG-1 and FWG-2 were best fitted to Korsmeyer plot $\left(R^{2} 0.974\right.$ and 0.967 respectively) with the $n$ value of 0.834 and 1.008 respectively. The release mechanism of DS from FWG-1 was mainly by diffusion and erosion whereas in case of FWG-2, complete zero order release (concentration independent) mechanism can be assumed. MDT is the indicator of sustaining action of a matrix tablet. FWG-1 and FWG-2 gave more sustained action, that's why they had the more MDT values, that is, 4.037 and 4.587 hours respectively compared to FDC-1 and FDC-2 (having MDT of 3.314 and 3.680 hours respectively).

Table 2: Kinetic parameters of DS release from different polymeric matrix tablets (caplet oval).

\begin{tabular}{|c|c|c|c|c|c|c|c|c|}
\hline \multirow{2}{*}{$\begin{array}{c}\text { Formulation } \\
\text { code }\end{array}$} & \multirow{2}{*}{$\begin{array}{c}\text { \% drug } \\
\text { release } \\
\text { after 8 hrs }\end{array}$} & \multicolumn{2}{|c|}{ Zero Order } & \multicolumn{2}{|c|}{ Higuchi } & \multicolumn{2}{c|}{ Korsmeyer } & \multirow{2}{*}{$\begin{array}{c}\text { MDT } \\
\text { (hrs) }\end{array}$} \\
\cline { 3 - 8 } & $\mathbf{K}_{\mathbf{0}}$ & $\mathbf{R}^{\mathbf{2}}$ & $\mathbf{K}_{\mathbf{H}}$ & $\mathbf{R}^{\mathbf{2}}$ & $\mathbf{n}$ & $\mathbf{R}^{\mathbf{2}}$ & \\
\hline FDC-1 & 90.136 & 10.79 & 0.956 & 33.32 & 0.982 & 0.734 & 0.977 & 3.314 \\
\hline FDC-2 & 83.294 & 10.20 & 0.957 & 33.45 & 0.979 & 0.761 & 0.978 & 3.680 \\
\hline FWG-1 & 77.924 & 9.93 & 0.954 & 30.48 & 0.9673 & 0.834 & 0.974 & 4.037 \\
\hline FWG-2 & 73.136 & 9.72 & 0.958 & 29.42 & 0.9445 & 1.008 & 0.967 & 4.587 \\
\hline
\end{tabular}

\section{CONCLUSION}

In this work DS matrix tablet was prepared by using different polymers at different percentages. The use of polyethylene glycol (PEG), Hydroxpropylmethylcellulose (HPMC) with Kollidon SR in the DS matrix tablet showed some distinct release characteristics. Due to high percentage of kollidon SR (30\%) and HPMC 15 cps, FDC-1 and FDC-2 showed less sustained release than FWG-1 and FWG-2 (containing 25\% Kollidon SR and PEG 600). Between the two manufacturing methods, direct compression found to release the drug more quickly than wet granulation which was evident from their MDT values. Again, among the three different shapes, caplet oval shaped tablets prepared in direct compression process released highest amount of DS than the two shapes. 
Ahmed et al., 2009

\section{REFERENCES}

Amaral MH, Lobo JMS, Ferreira DC. (2001) Effect of Hydroxypropyl methylcellulose and hydrogenated castor oil on naproxen release from sustained-release tablets. AAPS PharmSciTech. 2(2) Article 6.

BASF, Technical Information, ME 397e, June 1999.

Colombo P, Catellani PL, Peppas NA. (1992) Swelling characteristics of hydrophilic matrices for controlled release: new dimensionless number to describe the swelling and release behavior. Int. J. Pharm 88: 99-109.

Donbrow M, Samuelov, Y. (1980) Zero order drug delivery from double-layered porous films: release rate profiles from ethylcellulose, hydroxypropylcellulose and polyethylene glycol mixtures. J. Pharm. Pharmacol. 32: 463-470.

Higuchi T. (1961) Rate of release of medicaments from ointment bases containing drugs in suspension. J. Pharm. Sci. 50: 874-875.

Korsmeyer RW, Gurny R, Doelker EM, Buri P, Peppas NA. (1983) Mechanism of solute releasefrom porous hydrophilic polymers. Int. J. Pharm. 15: 25-35.

Lordi NG. (1990) Sustained release dosage forms. In: Lachman L, Lieberman HA, Kanig JL. Eds. The Theory and Practice of Industrial Pharmacy. Varghese Publishing House, Bombay. Edn. $3^{\text {rd }}$, pp.430-456.

Peppas NA. (1985) Analysis of Fickian and non-Fickian drug release from polymers. Pharm. Acta. Helv. 60: 110-111.

Reynolds TD, Mitchell SA, Balwinski KM. (2004) Investigation of the effect of tablet surface area/volume on drug release from hydroxypropylmethylcellulose controlled-release matrix tablets. Drug Dev. Ind. Pharm. 28(4): 457-466.

Salsa T, Veiga F, Pina ME. (1997) Oral controlled-release dosage forms. I. Cellulose ether polymers in hydrophilic matrices. Drug Dev. Ind. Pharm. 23:929-938. 